\title{
Learning Restricted Boltzmann Machines via Influence Maximization*
}

\author{
Guy Bresler \\ guy@mit.edu \\ Massachusetts Institute of \\ Technology. Dept. of Electrical Eng. \\ and Computer Science and IDSS. \\ Cambridge, MA, USA
}

\author{
Frederic Koehler \\ fkoehler@mit.edu \\ Massachusetts Institute of \\ Technology. Department of \\ Mathematics. \\ Cambridge, MA, USA
}

\author{
Ankur Moitra \\ moitra@mit.edu \\ Massachusetts Institute of \\ Technology. Department of \\ Mathematics, CSAIL and IDSS. \\ Cambridge, MA, USA
}

\begin{abstract}
Graphical models are a rich language for describing high-dimensional distributions in terms of their dependence structure. While there are algorithms with provable guarantees for learning undirected graphical models in a variety of settings, there has been much less progress in the important scenario when there are latent variables. Here we study Restricted Boltzmann Machines (or RBMs), which are a popular model with wide-ranging applications in dimensionality reduction, collaborative filtering, topic modeling, feature extraction and deep learning.

The main message of our paper is a strong dichotomy in the feasibility of learning RBMs, depending on the nature of the interactions between variables: ferromagnetic models can be learned efficiently, while general models cannot. In particular, we give a simple greedy algorithm based on influence maximization to learn ferromagnetic RBMs with bounded degree. In fact, we learn a description of the distribution on the observed variables as a Markov Random Field. Our analysis is based on tools from mathematical physics that were developed to show the concavity of magnetization. Our algorithm extends straighforwardly to general ferromagnetic Ising models with latent variables.

Conversely, we show that even for a contant number of latent variables with constant degree, without ferromagneticity the problem is as hard as sparse parity with noise. This hardness result is based on a sharp and surprising characterization of the representational power of bounded degree RBMs: the distribution on their observed variables can simulate any bounded order MRF. This result is of independent interest since RBMs are the building blocks of deep belief networks.
\end{abstract}

${ }^{*}$ G.B. was supported in part by ONR N00014-17-1-2147, and A.M was supported in part by NSF CAREER Award CCF-1453261, NSF Large CCF-1565235, a David and Lucile Packard Fellowship, an Alfred P. Sloan Fellowship and an ONR Young Investigator Award

Permission to make digital or hard copies of all or part of this work for personal or classroom use is granted without fee provided that copies are not made or distributed for profit or commercial advantage and that copies bear this notice and the full citation on the first page. Copyrights for components of this work owned by others than the author(s) must be honored. Abstracting with credit is permitted. To copy otherwise, or republish, to post on servers or to redistribute to lists, requires prior specific permission and/or a fee. Request permissions from permissions@acm.org.

STOC '19, fune 23-26, 2019, Phoenix, AZ, USA

(c) 2019 Copyright held by the owner/author(s). Publication rights licensed to ACM. ACM ISBN 978-1-4503-6705-9/19/06 ..\$15.00

https://doi.org/10.1145/3313276.3316372

\section{CCS CONCEPTS}

- Mathematics of computing $\rightarrow$ Markov networks; • Theory of computation $\rightarrow$ Sample complexity and generalization bounds; Unsupervised learning and clustering.

\section{KEYWORDS}

Graphical models, Restricted Boltzmann Machines, submodularity, unsupervised learning

\section{ACM Reference Format:}

Guy Bresler, Frederic Koehler, and Ankur Moitra. 2019. Learning Restricted Boltzmann Machines via Influence Maximization. In Proceedings of the 51st Annual ACM SIGACT Symposium on the Theory of Computing (STOC '19), June 23-26, 2019, Phoenix, AZ, USA. ACM, New York, NY, USA, 12 pages. https://doi.org/10.1145/3313276.3316372

\section{INTRODUCTION}

\subsection{Background}

Graphical models are a rich language for describing high-dimensional distributions in terms of their dependence structure. They allow for sophisticated forms of causal reasoning and inference. Over the years, many provable algorithms for learning undirected graphical models from data have been developed, including algorithms that work on trees [11], graphs of bounded treewidth [23], graphs of bounded degree [6, 16, 24, 39], and under various conditions on their parameters that preclude long-range correlations $[1,8,32]$. In the special case of Ising models, one can learn the underlying graph in nearly quadratic time with nearly the information-theoretically optimal sample complexity [24, 39].

While all these results are for fully observed models, the presence of unobserved (or latent) variables is of fundamental importance in a wide range of applications. Latent variable models can capture much more complex dependencies among the observed variables than fully observed models, because the variables can influence each other through unobserved mechanisms. In this way, such models allow scientific theories that explain data in a more parsimonious way to be learned and tested. They can also be used to perform dimensionality reduction [18] and feature extraction [12] and thus serve as a basis for a variety of other machine learning tasks.

Despite their practical importance, the problem of learning graphical models with latent variables has seen much less progress. The only works we are aware of are the following: Chadrasekaran et al. [10] studied Gaussian graphical models with latent variables and sparsity and incoherence constraints. The marginal distribution on the observed variables is also a Gaussian graphical model, so 
it is straightforward to learn its distribution. However their focus was on discovering latent variables whose inclusion in the model "explains away" many of the observed dependencies. Anandkumar and Valluvan [2] were the first to give provable algorithms for learning discrete graphical models with latent variables, although they need rather strong conditions to do so. They require both that the graphical model is locally treelike and that it exhibits correlation decay.

In this paper we study Restricted Boltzmann Machines (or RBMs), a widely-used class of graphical models with latent variables that were popularized by Geoffrey Hinton in the mid 2000s. In fact, our results will extend straightforwardly to general Ising models with latent variables. An RBM has $n$ observed (or visible) variables $X_{1}, X_{2}, \ldots, X_{n}$ and $m$ latent (or hidden) variables $Y_{1}, Y_{2}, \ldots, Y_{m}$ and is described by

(1) an $n \times m$ interaction matrix $J$

(2) a length $n$ vector $h^{(1)}$ and a length $m$ vector $h^{(2)}$ of external fields

Then for any $x \in\{ \pm 1\}^{n}$ and $y \in\{ \pm 1\}^{m}$, the probability that the model assigns to this configuration is given by:

$$
\operatorname{Pr}(X=x, Y=y)=\frac{1}{Z} \exp \left(x^{T} J y+\sum_{i=1}^{n} h_{i}^{(1)} x_{i}+\sum_{i=1}^{m} h_{j}^{(2)} y_{j}\right)
$$

where $Z$ is the partition function. It is often convenient to think about an RBM as a weighted bipartite graph whose nodes represent variables and whose weights are given by $J$. This family of models has found a number of applications including in collaborative filtering [34], topic modeling [19] and in deep learning where they are layered on top of each other to form deep belief networks [17]. As the number of layers grows, they can capture increasingly complex hierarchical dependencies among the observed variables.

We focus on the problem of learning RBMs from i.i.d. samples of the observed variables, with particular emphasis on the practically relevant case where the latent variables have low degree. What makes this challenging is that even though the variables in the RBM have only pairwise interactions, when the latent variables are marginalized out we can (and usually do) get higher-order interactions. Indeed, for general graphical models with latent variables and pairwise interactions, Bogdanov, Mossel and Vadhan [5] proved learning is hard (assuming $N P \neq R P$ ) by showing how the distribution on observed variables can simulate the uniform distribution on satisfying assignments of any given circuit. We note that this construction requires a large number (at least one for each gate) of interconnected latent variables and that the hard instances are highly complex because they come from a series of circuit manipulations. Beyond learning, Long and Servedio [27] proved that for RBMs a number of other related problems are hard, including approximating the partition function within an exponential factor and approximate inference and sampling.

The previous work leaves the following question unresolved: Are there natural and well-motivated families of Ising models with latent variables that can be efficiently learned? We will answer this question affirmatively in the case of ferromagnetic RBMs and (more generally) ferromagnetic Ising models with latent variables, which are defined as follows: A ferromagnetic RBM is one in which the interaction matrix and the vectors of external fields are nonnegative.
On the other hand, we give a negative result showing that without ferromagneticity, even in the highly optimistic case when there are only a constant number of latent variables with bounded degree the problem is as hard as sparse parity with noise. This establishes a dichotomy that is just not present in the fully-observed setting.

Historically, ferromagneticity is a natural and well-studied property that plays a key role in many classic results in statistical physics and theoretical computer science. For example, the Lee-Yang theorem [25] shows that the complex zeros of the partition function of a ferromagnetic Ising model all lie on the imaginary axis - this property does not hold for general Ising models. Ferromagnetic Ising models are also one of the largest classes of graphical models for which there are efficient algorithms for sampling and inference, which follows from the seminal work of Jerrum and Sinclair [21]. This makes them an appealing class of graphical models to be able to learn. In contrast, without ferromagneticity it is known that sampling and inference are computationally hard when the Gibbs measure on the corresponding infinite $d$-regular tree becomes non-unique [36].

\subsection{Our Results}

First we focus on learning ferromagnetic Restricted Boltzmann Machines with bounded degree. The idea behind our algorithm is simple: the observed variables that exert the most influence on some variable $X_{i}$ ought to be $X_{i}$ 's two-hop neighbors. While this may seem intuitive, the most straightforward interpretation of this statement is false - the variable with the largest correlation with $X_{i}$ may actually be far away. In addition, even if we correct the statement (e.g. by stating instead that there should be a neighbor with large influence), such facts about graphical models are often subtle and challenging to prove. Ultimately, we make use of the famous Griffiths-Hurst-Sherman correlation inequality [15] to prove that the discrete influence function

$$
I_{i}(S)=\mathrm{E}\left[X_{i} \mid X_{S}=\{+1\}^{|S|}\right]
$$

is submodular (see Theorem 3.6). The GHS inequality has found many applications in mathematical physics where it is an important ingredient in determining critical exponents at phase transitions. By recognizing that the concavity of magnetization is analogous to the properties of the multilinear extension of a submodular function [9], we are able to bring to bear tools from submodular maximization to learning graphical models with latent variables.

More precisely, we show that any set $T$ that is sufficiently close to being a maximizer of $I_{i}$ must contain the two-hop neighbors of $X_{i}$. We can thus use the greedy algorithm for maximizing a monotone submodular function [31] to reduce our problem of finding the two-hop neighbors of $X_{i}$ to a set of constant size, where the constant depends on the maximum degree and upper and lower bounds on the strength of non-zero interactions. It is information theoretically impossible to learn $J, h^{(1)}$ and $h^{(2)}$ uniquely, but we do something almost as good and learn a description of the distribution of the observed variables as a Markov Random Field (or MRF, see Definition 4.1):

THEOREM 1.1 (INFORMAL). There is a nearly quadratic time algorithm with logarithmic sample complexity for learning the distribution of observed variables (expressed as a Markov Random Field) for 
ferromagnetic Restricted Boltzmann Machines of bounded degree and upper and lower bounded interaction strength.

See Theorem 6.1 and Theorem 6.3 for the precise statement. We note that unlike earlier greedy algorithms for learning Ising models [6, 16] our dependence on the maximum degree is singly exponential and hence is nearly optimal [35]. In independent work, Lynn and Lee [28] also considered the problem of maximizing the influence but in a known Ising model. They gave a (conjecturally optimal) algorithm for solving this problem given an $\ell_{1}$-constraint on the external field.

Our algorithm extends straightforwardly to general ferromagnetic Ising models with latent variables. In this more general setting, the two-hop neighborhood of a node $i$ is replaced by an induced Markov blanket (see Definition 4.2), which informally corresponds to the set of observed nodes that separate $i$ from the other observed nodes. We prove:

THEOREM 1.2 (INFORMAL). There is a nearly quadratic time algorithm with logarithmic sample complexity for learning the distribution of observed variables (expressed as a Markov Random Field) for ferromagnetic Ising model with latent variables, under the conditions that the interaction strengths are upper and lower bounded, the induced Markov blankets have bounded size and that the distance between any node $i$ and any other node in its Markov blanket is bounded.

See Theorem 6.4 and Theorem 6.5 for the precise statement. We remark that in our setting, the maximal Fourier coefficients of the induced MRF can be arbitrarily small, which is a serious obstacle to directly applying existing algorithms for learning MRFs (see Example C.5). Our method also has the advantage of running in near-quadratic time whereas existing MRF algorithms would require runtime $n^{d_{H}+1}$, where $d_{H}$ is the maximum hidden degree ${ }^{1}$. We also show how Lee-Yang properties that hold for ferromagnetic Ising models [26] carry over to the induced MRFs in the presence of latent variables, which allows us to approximate the partition function and perform inference efficiently. See Theorem 7.1 for the precise statement. Compared to the previous settings where provable guarantees were known, ours is the first to work even when there are long range correlations.

As we alluded to earlier, being ferromagnetic turns out to be the key property in avoiding computational intractability. More precisely, we show a rather surprising converse to the well-known fact that marginalizing out a latent variable produces a higher-order interaction among its neighbors. We show that marginalizing out a collection of latent variables can produce any desired higher-order interaction among their neighbors.

Theorem 1.3 (InFormal). Every binary Markov Random Field of order $d_{H}$ can be expressed as the distribution on observed variables of a Restricted Boltzmann Machine, where the maximum degree of any latent node is at most $d_{H}$.

See Theorem 4.4 for the precise statement. Our approach to showing the equivalence between RBMs and MRFs is to show a non-zero correlation bound between the soft absolute value function that arises from marginalizing out latent variables and a parity function.

\footnotetext{
${ }^{1}$ The induced MRF has order $d_{H}$, so these methods (e.g. [24]) need to solve regression problems on polynomials of degree $d_{H}$.
}

We accomplish this through estimates of the Taylor expansion of special functions. With this in hand, we can match the largest degree terms in the energy function of an MRF and recurse.

Apart its usefulness in proving hardness, this result also resolves a basic question about the representational power of RBMs. Towards the goal of understanding deep learning, a number of recent works have shown depth separations in feed-forward neural networks $[13,33,37]$. They explicitly construct (or show that there exists) a function that can be computed by a depth $d+1$ feed-forward neural network of small size, but with depth $d$ would require exponential size. In fact, RBMs are the building block of another popular paradigm in deep learning: deep belief networks [17]. Towards understanding the representational power of RBMs, Martens et al. [29] showed that it is possible to approximately represent the uniform distribution on satisfying inputs to the parity function, and more generally any predicate depending only on the number of 1 s, using a dense RBM. In practice, sparse RBMs are desirable because their dependencies are easier to interpret. The above theorem exactly characterizes what distributions can be represented this way: They are exactly the bounded order MRFs.

In any case, what this means for our lower bound is that without ferromagneticity, even RBMs with a constant number of latent variables of constant degree inherits the hardness results of learning MRFs [7, 24], that in turn follow from the popular assumption that learning sparse parities with noise is hard. For comparison, the technique used in [29] seems insufficient for this reduction - their method can only build certain noiseless functions.

COROLlARY 1.4 (INFORMAL). If $k$-sparse noisy parity on $n$ bits is hard to learn in time $n^{o(k)}$, then it is hard to learn a representation of the distribution on $n$ observed variables (as any unnormalized function that can be efficiently computed) that is close to within total variation distance $1 / 3$ of a Restricted Boltzmann Machine where the maximum degree of any latent node is $d_{H}$ in time $n^{o\left(d_{H}\right)}$. This is true even if the number of hidden nodes in the RBM is promised to be constant w.r.t. $n$.

See Theorem 5.5 for the precise statement. Recall that it is impossible to learn the parameters of an RBM uniquely. Our result shows that learning merely a description of the distribution on the observed variables - i.e. a form of improper learning - is hard too, even for RBMs with only a constant number of hidden variables. In contrast, previous lower bounds were for graphical models with many more latent variables than observed variables [5]. At the time it seemed plausible that there were large classes of graphical models with latent variables that could be efficiently learnable. But in light of how simple our hard examples are, it seems difficult to imagine any other natural and well-motivated class of graphical models with latent variables (without ferromagneticity) that is also easy to learn.

\subsection{Further Discussion}

There is an intriguing analogy between our results and the problem of learning juntas $[30,38]$. While the general problem of learning $k$-juntas seems to be hard to solve in time $n^{o(k)}$ there are some special cases that can be solved much faster. Most notably, if the junta is monotone then there is a simple algorithm that works: Find all the coordinates with non-zero influence and solve the junta 
learning problem restricted to those coordinates. We can think of ferromagneticity as the natural analogue of monotonicity in the context of RBMs, since this property also prevents certain types of cancellations. Are there other contraints that one can impose on RBMs, perhaps inspired by ones that work for juntas, that make the problem much easier?

Another enticing question for future work is to study "deeper" versions of the problem, such as ferromagnetic deep belief networks. Are there new provable algorithms for classes of deep networks to be discovered? There is a growing literature on learning deep networks under various assumptions [3, 14, 20, 40], but the ability of ferromagnetic RBMs to express long-range correlations seems to make it a potentially more challenging problem to tackle.

\section{PRELIMINARIES}

Definition 2.1. An Ising model is a probability distribution $\mu=$ $\mu(J, h)$ on the hypercube $\{ \pm 1\}^{n}$ under which

$$
\operatorname{Pr}(X=x)=\mu(x)=\frac{1}{Z} \exp \left(\frac{1}{2} \sum_{i, j} J_{i j} x_{i} x_{j}+\sum_{i} h_{i} x_{i}\right)
$$

where $J$ is a symmetric matrix with zero diagonal referred to as the interaction matrix, $h \in \mathbb{R}^{n}$ is the external field and normalizing constant $Z$ is the partition function.

Definition 2.2. A ferromagnetic Ising model (with consistent external fields) is an Ising model such that $J_{i j} \geq 0$ for all $i, j$ and such that $h_{i} \geq 0$. We will refer to such a $J$ as a ferromagnetic interaction matrix.

Clearly the joint distribution of a Restricted Boltzmann Machine is just a special case of a general Ising model. Therefore we say a Restricted Boltzmann Machine is ferromagnetic if $J_{i j} \geq 0, h_{i}^{(1)} \geq$ $0, h_{i}^{(2)} \geq 0$ which is consistent with our previous terminology.

\section{SUBMODULARITY OF INFLUENCE IN ISING MODELS}

Definition 3.1. Fix a ferromagnetic interaction matrix $J$. We define the smooth influence function for $X_{i}$ to be $\mathcal{I}_{i}(h)=\mathrm{E}_{X \sim \mu(J, h)}\left[X_{i}\right]$.

Definition 3.2. Suppose $f: \mathbb{R}_{\geq 0}^{n} \rightarrow \mathbb{R}$ is a $C^{2}$ function, i.e. it has continuous second partial derivatives. We say that $f$ is a smooth monotone submodular function if $\partial_{i} f \geq 0$ everywhere and $\partial_{i} \partial_{j} f \leq$ 0 everywhere for all $i, j$.

We will see that smooth monotone submodularity of $\mathcal{I}_{i}$ in ferromagnetic Ising models follows from the following correlation inequality of [15]:

Theorem 3.3 (GHS inequality, [15]). Let $J$ be the interaction matrix of a ferromagnetic Ising model on $n$ nodes without external field. Then for any (not necessarily distinct) $1 \leq i, j, k, \ell \leq n$ we have

$$
\begin{aligned}
& \mathbf{E}\left[X_{i} X_{j} X_{k} X_{\ell}\right]-\mathbf{E}\left[X_{i} X_{j}\right] \mathbf{E}\left[X_{k} X_{\ell}\right]-\mathbf{E}\left[X_{i} X_{k}\right] \mathbf{E}\left[X_{j} X_{\ell}\right] \\
& -\mathbf{E}\left[X_{i} X_{\ell}\right] \mathbf{E}\left[X_{j} X_{k}\right]+2 \mathbf{E}\left[X_{i} X_{\ell}\right] \mathbf{E}\left[X_{j} X_{\ell}\right] \mathbf{E}\left[X_{k} X_{\ell}\right] \leq 0,
\end{aligned}
$$

where the expectations are taken with respect to the Boltzmann distribution.

COROLlary 3.4. Let $J$ be a ferromagnetic interaction matrix, i.e. $J_{i j} \geq 0$. Then for any $i \in[n], \mathcal{I}_{i}(h): \mathbb{R}_{\geq 0}^{n} \rightarrow \mathbb{R}$ is a smooth monotone submodular function.
Corollary 3.4 follows from the well-known equivalence of correlation inequalities and partial derivative inequalities; for completeness, we include a proof in Appendix A. From this we deduce the below (discrete) submodularity result:

Definition 3.5. Fix a ferromagnetic Ising model $(J, h)$. We define the discrete influence function for $X_{i}$ to be a function from $S \subset[n]$ to $\mathbb{R}$ given by

$$
I_{i}(S)=\underset{X \sim \mu(J, h)}{\mathbf{E}}\left[X_{i} \mid X_{S}=\{+1\}^{|S|}\right]=\underset{X \sim \mu\left(J, h+\infty 1_{S}\right)}{\mathbf{E}}\left[X_{i}\right] .
$$

THEOREM 3.6. Fix a ferromagnetic Ising model $(J, h)$. Then for every $i$, the discrete influence function $I_{i}(S)$ is a monotone submodular function.

This submodularity has the following standard consequence, which will be very useful later.

Lemma 3.7. Fix a ferromagnetic Ising model $(J, h)$. Suppose $i \in[n]$ and $S, T \subset[n]$, and $I_{i}(T)>I_{i}(S)$. Then there exists $j \in T$ such that

$$
I_{i}(S \cup\{j\})-I_{i}(S) \geq \frac{I_{i}(T)-I_{i}(S)}{|T \backslash S|}
$$

\section{INTERREDUCIBILITY BETWEEN RBMS AND MRFS}

First we define (binary) Markov Random Fields and introduce some standard terminology:

Definition 4.1. A Markov Random Field (or MRF) of order $r$ is a probability distribution on $\{ \pm 1\}^{n}$ such that $\operatorname{Pr}(X=x)=\frac{1}{Z} \exp (f(x))$ where $f$ is a multivariate polynomial of degree $r$ such that $f(0)=0$, referred to as the potential. The structure graph of a Markov random field has vertices $1, \ldots, n$ and connects vertex $i$ and $j$ if there is a monomial in $f(x)$ involving both $x_{i}$ and $x_{j}$.

We will mostly be interested in Markov random fields of bounded degree, where the degree is a number typically larger than the order:

Definition 4.2. The degree of vertex $i$ in an MRF is its degree in the structure graph. We will refer to the maximum degree of all vertices as the degree of the MRF (structure graph).

Now we observe that the marginal distribution on the observable variables of a Restricted Boltzmann machine is a Markov Random Field, of order at most the max degree of a hidden node ${ }^{2}$. This is well known and was used for instance in [29].

Lemma 4.3. Fix a Restricted Boltzmann Machine $\left(J, h^{(1)}, h^{(2)}\right)$. Let $w_{j}$ be the $j^{\text {th }}$ column of J, i.e. the edge weights into hidden unit $j$. Then $P(X=x)=\frac{1}{Z} e^{f(x)}$ where $f(x)=\sum_{j=1}^{m} \rho\left(w_{j} \cdot x+h_{j}^{(2)}\right)+\sum_{i=1}^{n} h_{i}^{(1)} x_{i}$, $Z$ is the same as the partition function of the original $R B M$ and $\rho(x)=$ $\log \left(e^{x}+e^{-x}\right)$.

Our main result in this section is a reduction in the other direction: We show that every MRF can be converted to an equivalent Restricted Boltzmann Machine. This is more difficult and to our knowledge was not known before. We show we can build hidden

\footnotetext{
${ }^{2}$ This follows from the statement below because $\rho\left(w_{j} \cdot x+h_{j}^{(2)}\right)$ is a function on the relevant coordinates of $x$, and we represent it as a polynomial via its Fourier expansion on the corresponding hypercube.
} 
units with any desired correlation with a parity as long as the desired correlation is small - building many such units, we capture the MRF potential exactly.

THeORem 4.4. Consider an arbitrary order $r$ Markov random field inducing a probability distribution on the hypercube $\{ \pm 1\}^{n}$. Suppose that the structure graph of the MRF has degree $d$ and the coefficients of the potential $f$ are bounded by a constant $M$. There is an RBM with $n$ observable nodes and parameters $\left(J, h^{(1)}, h^{(2)}\right)$ with the following properties:

(1) The induced MRF of the RBM equals the original MRF, i.e. the marginal law of the observed variables is the same as the distribution of the original MRF.

(2) There are at most $O_{d, M}(n)$ hidden units $^{3}$, and the degree of every vertex in the hidden layer is at most $r$.

(3) The two-hop neighborhood of every observed node equals its original MRF-neighborhood. In particular the two-hop degree $d_{2}$ equals the degree d of the MRF (structure graph).

\section{THE LEARNING PROBLEM FOR RBMS}

Recall that we are interested in the problem of learning an RBM given samples from its marginal distribution on the observed nodes $X$ (but without observing hidden nodes $Y$ ). In our setting it is impossible to recover the underlying interaction matrix $J$ because it is not uniquely determined, i.e. RBMs are over-parameterized. We give a number of examples illustrating this in Appendix C.1.

Instead, we consider the natural objectives from the perspective of viewing the observable distribution as a Markov Random Field: structure learning and learning the parameters of the Markov random field. We start with structure learning, which can be viewed as the problem of learning the two-hop neighborhoods of the observed random variables - i.e. learning the square of the adjacency matrix of the bipartite structure graph.

Definition 5.1. Let $i$ be an observed node in an $\operatorname{RBM}\left(J, h^{(1)}, h^{(2)}\right)$. The two-hop neighborhood of $i$, denoted $\mathcal{N}_{2}(i)$, is the smallest $\operatorname{set}^{4} S \subset$ $[n] \backslash\{i\}$ such that conditioned on $X_{S}, X_{i}$ is conditionally independent of $X_{j}$ for all $j \in[n] \backslash(S \cup\{i\})$. The two-hop degree $d_{2}$ of an $\mathrm{RBM}$ is the maximum size of $\mathcal{N}_{2}(i)$ over all observed nodes $i$.

Observe that $\mathcal{N}_{2}(i)$ is always a subset of the graph-theoretic twohop neighborhood of $i$, i.e. the smallest set $S$ such that vertex $i$ is separated from the other observable nodes in the structure graph of the RBM. However it may be a strict subset, as in Example C.1.

In order to learn the two-hop structure of an RBM it will be necessary to have lower and upper bounds on the edge weights of the model, so we introduce the following notion of degeneracy. This is a standard assumption in the literature on learning Ising models [6, 24, 39]. In particular, a lower bound is needed because otherwise it would be impossible to distinguish a non-edge from an edge with an arbitrarily weak interaction. An upper bound is needed to ensure the distribution of any variable is not arbitrarily close to being deterministic.

\footnotetext{
${ }^{3}$ Also, from the construction we see that few hidden units are used if few Fourie coefficients are nonzero.

${ }^{4}$ Note that $S$ is uniquely determined; it is the neighborhood of $i$ in the induced MRF.
}

Definition 5.2. We say that an Ising model is $(\alpha, \beta)$-nondegenerate ${ }^{5}$ if both:

(1) For every $i, j$ such that $\left|J_{i j}\right| \neq 0$, we have $\left|J_{i j}\right|>\alpha$.

(2) $\sum_{j}\left|J_{i j}\right|+\left|h_{i}\right| \leq \beta$ for every node $i$.

An $\mathrm{RBM}$ is $(\alpha, \beta)$-nondegenerate if it is $(\alpha, \beta)$-nondegenerate as an Ising model.

Though our learning algorithm will succeed under the above nondegeneracy assumption, as we show in Appendix C.2 this condition does not suffice for previous regression-based results to guarantee success with a similar number of samples.

\subsection{Hardness for Improperly Learning RBMs}

In this subsection we show that structure learning for general (non-ferromagnetic) RBMs takes time $n^{\Omega\left(d_{H}\right)}$ under the conjectured hardness for learning sparse parity with noise. Throughout only this section we use the terminology with high probability to refer to an event which occurs with probability going to zero as $n \rightarrow \infty$ faster than inverse-polynomial, i.e. asymptotically faster than $1 / f(x)$ for any polynomial $f$.

Definition 5.3. The $k$-sparse parity with noise distribution is the following distribution on $(X, Y)$ parameterized by $\eta \in(0,1 / 2)$ and an unknown subset $S$ of size at most $k$ :

(1) Sample $X \sim \operatorname{Unif}\left(\{-1,+1\}^{n}\right)$.

(2) With probability $1 / 2+\eta$, set $Y=\prod_{s \in S} X_{s}$, and with probability $1 / 2-\eta$, set $Y=(-1) \prod_{s \in S} X_{s}$.

The learning problem for $k$-sparse parity with noise is to learn $S$ in polynomial time with high probability, given access to an oracle which generates samples of $(X, Y)$.

The important point is that the joint distribution of an $(r-1)$ sparse parity with noise $(X, Y)$ is a Markov Random Field with order $r$ interactions, and by Theorem 4.4 it is also the marginal distribution on the observables of an MRF with maximum hidden degree $d_{H}$, where the two-hop neighborhood of $Y$ is exactly the set $S$. This means if we could learn the two-hop neighborhoods of an RBM in time $n^{o\left(d_{H}\right)}$ this would yield a $n^{o(k)}$ algorithm for learning $k$-sparse parities with noise, which is a long-standing open question in theoretical computer science and conjectured to be impossible. The best known algorithm of Valiant [38] runs in time $n^{0.8 k}$. We summarize this observation as follows:

OBSERVATION 1. If $k$-sparse parity with noise on $n$ bits cannot be learned in time $n^{o(k)}$, then there is no algorithm which runs in time $n^{o\left(d_{H}\right)}$ and learns the two-hop neighborhood structure of a general $R B M$ from samples of the distribution on its observed nodes.

Remark 5.4. We see from the proof of Theorem 4.4 that only a constant number of hidden nodes (in terms of $n$ ) are used in the construction of the sparse parity RBM, so the above result holds even if the RBM is promised to have $O_{d_{H}}(1)$ many hidden nodes.

We will now furthermore show that this result applies even in the case of improper learning, where we do not aim to learn the structure but instead aim to learn a different distribution close to

\footnotetext{
${ }^{5}$ The notational convention differs from [6], where $\beta$ denotes the maximum edge weight.
} 
the RBM. In fact, the hardness result extends even to the case when we wish to learn only an unnormalized probability mass function.

THEOREM 5.5. If $k$-sparse parity with noise on $n$ bits cannot be learned in time $n^{o(k)}$, then there is no algorithm which runs in time $n^{o\left(d_{H}\right)} \cdot \operatorname{poly}(1 / \epsilon)$ and returns a probability distribution $Q$ such that:

(1) $\|Q-\mathcal{P}\|_{\mathrm{TV}}<\epsilon$ where $\mathcal{P}$ is the distribution on the observables of an $R B M$ with hidden degree $d_{H}$.

(2) There exists a function $q(x, y)$ such that $Q(x, y)=\frac{1}{C_{q}} q(x, y)$ and $q(x, y)$ is efficiently (approximately) computable.

\section{A GREEDY ALGORITHM FOR LEARNING FERROMAGNETIC RBMS}

We describe a simple and efficient greedy algorithm for learning the two-hop neighborhood of an observed node $i$ from samples, if the RBM is ferromagnetic. This algorithm is much faster than is possible for general RBMs according to the lower bound of the previous subsection. Let $\widetilde{\mathbf{E}}$ denote the empirical expectation, and define the empirical influence

$$
\widetilde{I_{i}}(S)=\widetilde{\mathbf{E}}\left[X_{i} \mid X_{S}=\{1\}^{S}\right] .
$$

Let $\eta>0$ be a real-valued parameter and $k \geq 1$ an integer parameter to be specified later.

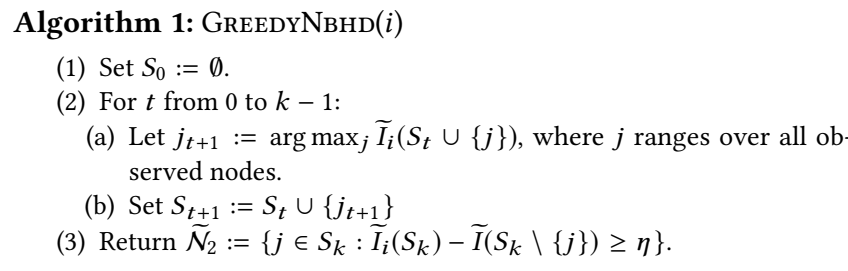

Theorem 6.1. Let $\delta>0$. Suppose $X^{(1)}, \ldots, X^{(M)}$ are samples from the observable distribution of a ferromagnetic Restricted Boltzmann machine which is $(\alpha, \beta)$-nondegenerate, and has two-hop degree $d_{2}$. Then if

$$
M \geq 2^{2 k+3}\left(d_{2} / \eta\right)^{2}(\log (n)+k \log (e n / k)) \log (4 / \delta)
$$

where we set $\eta=\alpha^{2} \sigma(-2 \beta)(1-\tanh (\beta))^{2}$ and $k=d_{2} \log (4 / \eta)$ for every $i$ algorithm GREEDYNBHD returns $\mathcal{N}_{2}(i)$, with probability at least $1-\delta$. Furthermore the total runtime is $O\left(M k n^{2}\right)=$ $e^{O\left(\beta d_{2}-\log (\alpha)\right)} n^{2} \log (n)$.

We defer the complete proof of Theorem 6.1, which crucially uses the submodularity of influence from Theorem 3.6, to the appendix.

\subsection{Improving the Sample Complexity}

In the full version of this paper, we describe an algorithm which has better sample complexity than the previous one, but sacrifices speed in order to achieve this: it runs in time $O\left(n^{d_{2}+1} \log (n)\right)$. This leaves open the question of whether there is a statistical-computational gap inherent in the ferromagnetic RBM-learning problem.

TheOREM 6.2. Algorithm SEARCHNBHD returns the correct neighborhood with probability at least $1-\delta$ given $M \geq 2^{2 d_{2}+3}(1 / \eta)^{2}(\log (n)+$ $d_{2} \log \left(\right.$ en $\left.\left./ d_{2}\right)\right) \log (4 / \delta)$ samples, when $\eta=\alpha^{2} \sigma(-2 \beta)(1-\tanh (\beta))^{2}$. The algorithm runs in time $O\left(n^{d_{2}+1} M\right)$.

\subsection{Learning the Induced MRF via Regression}

Once the two-hop neighborhoods of the observed nodes in the RBM are determined, it becomes much easier to learn the potential (i.e. the hyper-edge weights) of the induced MRF; this is because the problem of predicting $X_{i}$ based on the other spins goes from being a high-dimensional regression problem in $O\left(n^{d_{H}}\right)$ monomials to a low-dimensional problem - we can restrict to the monomials supported on the actual two-hop neighborhood.

Our algorithm uses GREEDYNBHD to learn the two-hop neighborhoods of the MRF, combined with running low-dimensional regressions ${ }^{6}$ within each of these neighborhoods to learn the coefficients of the MRF potential, and we show it successfully reconstructs the potential $p^{*}$. Here we adopt the convention that the constant term of the potential $p^{*}$ is 0 , since it is otherwise ambiguous.

THEOREM 6.3. Consider an unknown $(\alpha, \beta)$-nondegenerate ferromagnetic Restricted Boltzmann Machine with two-hop degree $d_{2}$. Let $p^{*}(x)$ be the potential of the MRF induced on the observed nodes. There exists an algorithm, which given $\alpha, \beta, d_{2}$, with probability at least $1-\delta$ finds a polynomial $p$ of degree at most $d_{2}$ such that

$$
\left\|\hat{p}-p^{*}\right\|_{\infty}^{2}=O\left(\frac{\beta \sigma(-2 \beta)^{d_{2}}}{2^{d_{2} / 2}\left(1-\tanh ^{2}(\beta)\right)^{2}} \sqrt{\frac{\log (M n / \delta)}{M}}\right)
$$

given $M$ samples from the the distribution on the observed nodes, provided that $M$ is at the required $M$ in Theorem 6.1.

In the full version of the paper, we give an example showing that a bounded-degree assumption is necessary for a result like Theorem 6.3 to hold, even without hidden nodes in the model. We also note that under a certain "anti-laminar" condition Theorem 6.3 enables us to recover the location of hidden nodes in the RBM.

\subsection{Learning Ferromagnetic Ising Models with Arbitrary Latent Variables}

In the full version of this paper, we show how all of the above results generalize beyond the RBM setting to ferromagnetic Ising models with an arbitrary set of hidden nodes - where the interaction matrix can connect pairs of observed nodes and pairs of hidden nodes too.

TheOREM 6.4. Let $\delta>0$. Suppose $X^{(1)}, \ldots, X^{(M)}$ are samples from the observable distribution of a ferromagnetic Ising model with hidden nodes which is $(\alpha, \beta)$-nondegenerate. Suppose that for every $i$ and $j \in \mathcal{N}_{2}(i)$, there is a path of length at most $\ell$ from $i$ to $j$. If

$$
M \geq 2^{2 k+3}\left(d_{2} / \eta\right)^{2}(\log (n)+k \log (e n / k)) \log (4 / \delta)
$$

where we set $\eta=\alpha^{\ell} \sigma(-2 \beta)(1-\tanh (\beta))^{\ell}$ and $k=d_{2} \log (4 / \eta)$, then algorithm GREEDYNBHD returns $\mathcal{N}_{2}(i)$ correctly for every $i$, with probability at least $1-\delta$. Furthermore the total runtime is $O\left(M k n^{2}\right)=$ $e^{O\left(\beta \ell d_{2}-\ell \log (\alpha)\right)} n^{2} \log (n)$.

Theorem 6.5. Consider an unknown $(\alpha, \beta)$-nondegenerate Ising model with hidden nodes and $d_{2}, \ell$ as in statement of Theorem 6.4. Let $p^{*}(x)$ be the potential of the MRF induced on the observed nodes. There exists an algorithm, which given $\alpha, \beta, d_{2}, \ell$, with probability at

\footnotetext{
${ }^{6}$ we use the GLMTron algorithm of [22] but many other methods work
} 
least $1-\delta$ finds a polynomial p of degree at most $d_{2}$ such that

$$
\left\|\hat{p}-\hat{p}^{*}\right\|_{\infty}^{2}=O\left(\frac{\beta \sigma(-2 \beta)^{d_{2}}}{2^{d_{2} / 2}\left(1-\tanh ^{2}(\beta)\right)^{2}} \sqrt{\frac{\log (M n / \delta)}{M}}\right)
$$

given $M$ samples from the distribution on observed nodes provided that $M$ is at the required $M$ in Theorem 6.4.

\section{INFERENCE ON THE INDUCED MRF VIA THE LEE-YANG PROPERTY}

The following theorem builds upon results from [26], which is based on Barvinok's approach [4] for approximating the partition function. We defer all details of the proof to the full version of the paper; the key insight is that the crucial Lee-Yang property [25] of the ferromagnetic Ising model is inherited by the MRF which arises from marginalizing out hidden nodes.

Theorem 7.1. Fix $H>0$ and a maximum degree $d_{2}$. Then for any ferromagnetic RBM in which $h_{i}^{(1)} \leq-H$ for all $i$, there is a deterministic polynomial time algorithm which given any $0<\epsilon<$ $1 / 4$ and the description of the induced MRF, computes $\log Z$ within additive error $\epsilon / 4$ where $Z$ is the partition function of the induced $M R F$.

The significance of accurately estimating $\log Z$ is that it allows for the performance of various inference tasks which are otherwise computationally intractable. For example, we can estimate to high precision the likelihood of observing any particular output from the MRF, since $\log \operatorname{Pr}(X=x)=p(x)-\log Z$ where $p(x)$ is the potential of the MRF. Hence the $\epsilon / 4$ approximation to $\log Z$ from Theorem 7.1 implies an $\epsilon / 4$ approximation to $\log \operatorname{Pr}(X=x)$, i.e. a PTAS for estimating $\operatorname{Pr}(X=x)$.

\section{A APPENDIX: OMITTED PROOFS FROM SEC 3}

Proof of Corollary 3.4. The equivalence of correlation inequalities and partial derivative inequalities is well-known (and is used in [15]); we include a proof only for completeness, since this precise statement does not appear in [15].

Let $Z(h)$ denote the partition function of the Ising model with interaction matrix $J$ and external field $h$. Then observe that

$$
\mathcal{I}_{i}(h)=\frac{\sum_{x} x_{i} \exp \left(x^{T} J x+h \cdot x\right)}{Z(h)}=\partial_{i} \log Z(h),
$$

so it suffices to prove that $\partial_{j} \partial_{i} \log Z(h) \geq 0$ for all $i, j$ and also that $\partial_{k} \partial_{j} \partial_{i} \log Z(h) \leq 0$ for all $i, j, k$. First observe by computing partial derivatives that

$$
\partial_{j} \partial_{i} \log Z(h)=\operatorname{Cov}\left(X_{i}, X_{j}\right) \geq 0,
$$

where the covariance is taken with respect to $\mu(J, h)$ and the inequality follows from Griffiths inequality. One can similarly observe that

$$
\begin{aligned}
& \partial_{k} \partial_{j} \partial_{i} \log Z(h)= \\
& \quad \mathrm{E}\left[X_{i} X_{j} X_{k}\right]-\mathrm{E}\left[X_{i} X_{k}\right] \mathrm{E}\left[X_{j}\right]-\mathrm{E}\left[X_{i}\right] \mathrm{E}\left[X_{j} X_{k}\right]-\mathrm{E}\left[X_{i} X_{j}\right] \mathrm{E}\left[X_{k}\right] \\
& \quad+2 \mathrm{E}\left[X_{i}\right] \mathrm{E}\left[X_{j}\right] \mathrm{E}\left[X_{k}\right],
\end{aligned}
$$

where the expectation is taken with respect to $\mu(J, h)$. We now eliminate the external field by the introduction of a ghost vertex
$X_{n+1}$ such that in the new Ising model, $J_{i(n+1)}=h_{i}, J_{i j}$ is otherwise the same as before and there is no external field. In this new Ising model the marginal of $X_{1}, \ldots, X_{n}$ given $X_{n+1}=1$ is the same as their distribution in the first Ising model, and the marginal given $X_{n+1}=-1$ is the same but with flipped signs. Letting $\mathbf{E}_{v}$ denote expectation with respect to this new Ising model, we see that

$$
\begin{aligned}
& \mathbf{E}\left[X_{i} X_{j} X_{k}\right]-\mathbf{E}\left[X_{i} X_{k}\right] \mathbf{E}\left[X_{j}\right]-\mathbf{E}\left[X_{i}\right] \mathbf{E}\left[X_{j} X_{k}\right]-\mathbf{E}\left[X_{i} X_{j}\right] \mathbf{E}\left[X_{k}\right] \\
& \quad+2 \mathbf{E}\left[X_{i}\right] \mathbf{E}\left[X_{j}\right] \mathbf{E}\left[X_{k}\right] \\
& =\underset{v}{\mathbf{E}}\left[X_{i} X_{j} X_{k} X_{\ell}\right]-\underset{v}{\mathbf{E}}\left[X_{i} X_{j}\right] \underset{v}{\mathbf{E}}\left[X_{k} X_{\ell}\right]-\underset{v}{\mathbf{E}}\left[X_{i} X_{k}\right] \underset{v}{\mathbf{E}}\left[X_{j} X_{\ell}\right] \\
& \quad-\underset{v}{\mathbf{E}}\left[X_{i} X_{\ell}\right] \underset{v}{\mathbf{E}}\left[X_{j} X_{k}\right]+2 \underset{v}{\mathbf{E}}\left[X_{i} X_{\ell}\right] \underset{v}{\mathbf{E}}\left[X_{j} X_{\ell}\right] \underset{v}{\mathbf{E}}\left[X_{k} X_{\ell}\right],
\end{aligned}
$$

where $\ell=n+1$. Thus it suffices to verify that this last expression is at most zero, which follows from Theorem 3.3.

Proof of Theorem 3.6. Since $I_{i}(S)=\mathbf{E}_{\mu\left(J, h+\infty 1_{S}\right)}\left[X_{i}\right]$, monotonicity follows immediately from Corollary 3.4. Similarly, submodularity follows because if $S \subset T$ and we let $h_{S}=h+\infty \cdot 1_{S}$ and likewise for $h_{T}$, then we obtain

$$
\begin{aligned}
I_{i}(S \cup\{j\})-I_{i}(S) & =\int_{h_{j}^{\prime}=0}^{\infty} \partial_{j} \mathcal{I}_{i}\left(h_{S}+h_{j}^{\prime} e_{j}\right) \\
& \geq \int_{h_{j}^{\prime}=0}^{\infty} \partial_{j} \mathcal{I}_{i}\left(h_{T}+h_{j}^{\prime} e_{j}\right)=I_{i}(T \cup\{j\})-I_{i}(T),
\end{aligned}
$$

where the inequality follows point-wise, by integrating the inequality $\partial_{k} \partial_{j} \mathcal{I}_{i} \leq 0$ along any coordinate-wise non-decreasing path from $h_{S}+h_{j}^{\prime} e_{j}$ to $h_{T}+h_{j}^{\prime} e_{j}$.

Proof of Lemma 3.7. This follows because

$$
I_{i}(S \cup T)-I_{i}(S) \geq I_{i}(T)-I_{i}(S)
$$

and by submodularity, since we can go from $S$ to $S \cup T$ by adjoining elements of $T \backslash S$ one-by-one,

$$
\begin{aligned}
I_{i}(S \cup T)-I_{i}(S) & \leq \sum_{j \in T \backslash S} I_{i}(S \cup\{j\})-I_{i}(S) \\
& \leq|T \backslash S| \max _{j \in T \backslash S}\left(I_{i}(S \cup\{j\})-I_{i}(S)\right)
\end{aligned}
$$

which completes the proof.

\section{B APPENDIX TO SECTION 4}

\section{B.1 Proof of Theorem 4.4}

The key technical fact underlying Theorem 4.4 is the following lemma, which shows that we can build an RBM with hidden nodes connected to the observed nodes in the set $S$ with any desired correlation with a parity on $S$ as long as the desired correlation is small.

Lemma B.1. Fix $k \geq 0$ and let $\rho(x)=\log \left(e^{x}+e^{-x}\right)$. Then there exist constants $\delta=\delta(k)>0$ and $\gamma=\gamma(k) \in(0, \pi / 2)$ such that for any $\delta^{\prime}$ with $\left|\delta^{\prime}\right|<\delta$ and $S \subset[n]$ with $|S|=k$, there exist $w, h$ with $|w|_{1}+h \leq \gamma$ such that

$$
\underset{X \sim\{ \pm 1\}^{n}}{\mathbf{E}}\left[\rho\left(w \cdot X_{S}+h\right) \chi_{S}(X)\right]=\delta^{\prime}
$$

where the expectation is with respect to uniform measure. 
Proof of Lemma 4.3. We show a slightly more general fact. Consider a general Markov Random Field of the form $\operatorname{Pr}(X=x)=$ $\frac{1}{Z} \exp (f(x))$ where $u$ is a vertex with only pairwise interactions, i.e.

$$
f(x)=h_{u} x_{u}+\sum_{v \sim u} w_{u v} x_{u} x_{v}+g\left(x_{\sim u}\right) .
$$

We now compute the marginal distribution on the model when $u$ is hidden. Observe that

$\operatorname{Pr}\left(X_{\sim u}=x_{\sim u}\right)=\exp \left(g\left(x_{\sim u}\right)\right) \frac{\sum_{x_{u}} \exp \left(h_{u} x_{u}+\sum_{v \sim u} w_{u v} x_{u} x_{v}\right)}{Z}$

so if we let $U$ denote the neighborhood of $u$ and let

$$
f_{U}\left(x_{U}\right)=\log \sum_{x_{u}} \exp \left(h_{u} x_{u}+\sum_{v \sim u} w_{u v} x_{u} x_{v}\right)=\rho\left(h_{u}+\sum_{v} w_{u v} \cdot x_{v}\right)
$$

where $\rho(x)=\log \left(e^{x}+e^{-x}\right)$ then

$$
\operatorname{Pr}\left(X_{\sim u}=x_{\sim u}\right)=\frac{\exp \left(g\left(x_{\sim u}\right)+f_{U}\left(x_{U}\right)\right)}{Z}
$$

Applying this inductively gives the result of the lemma.

Proof of Lemma B.1. This will follow by using the explicit formula for the taylor expansion of $\rho(x)$, which we will now derive. Recall $\rho^{\prime}(x)=\tanh (x)$ and that tanh has an explicit power series expansion with radius $\pi / 2$ around 0 :

$$
\tanh (x)=\sum_{n=1}^{\infty} \frac{2^{2 n}\left(2^{2 n}-1\right) B_{2 n}}{(2 n) !} x^{2 n-1}
$$

with radius of convergence $\pi / 2$, where $B_{2 n}=\frac{(-1)^{n+1} 2(2 n !)}{(2 \pi)^{2 n}} \zeta(2 n)$ are the even Bernoulli numbers. By integrating, we see

$$
\rho(x)=\log 2+\sum_{n=1}^{\infty} \frac{2^{2 n}\left(2^{2 n}-1\right) B_{2 n}}{(2 n) !(2 n)} x^{2 n}
$$

with the same radius of convergence.

We will need the standard fact that $B_{2 n} \neq 0$ for any $n \geq 1$, which follows immediately from the equation $B_{2 n}=\frac{(-1)^{n+1} 2(2 n !)}{(2 \pi)^{2 n}} \zeta(2 n)$ and the fact that $\zeta(s)=\sum_{m=1}^{\infty} \frac{1}{m^{s}}>0$ for $s>1$.

Now we use that the Fourier expansion of $\rho\left(w \cdot X_{S}+h\right)$ can be found by taking the power series expansion of $\rho$, plugging in $x=w \cdot X_{S}+h$ and using the identity $X_{i}^{2}=1$ to reduce to the parity basis. Let $m=\left\lceil\frac{|S|}{2}\right\rceil$ and take $\gamma \in(0, \pi / 2)$. By restricting to $w, h$ such that $|w|_{1}+|h|<\gamma$ we can write

$\rho\left(w \cdot X_{S}+h\right)=\log 2+\sum_{n=1}^{m} \frac{2^{2 n}\left(2^{2 n}-1\right) B_{2 n}}{(2 n) !(2 n)}\left(w \cdot X_{S}+h\right)^{2 n}+O\left(\gamma^{2 m+2}\right)$.

Note that in the sum, only the top $n=m$ term contributes to the coefficient of $\chi_{S}$. Observe that when $|S|$ is even ${ }^{7}$,

$$
\left[\chi_{S}\right]\left(w \cdot X_{S}+h\right)^{2 m}=|S| ! \prod_{s \in S} w_{S}
$$

and when $|S|$ is odd

$$
\left[\chi_{S}\right]\left(w \cdot X_{S}+h\right)^{2 m}=|S| ! h \prod_{s \in S} w_{S} .
$$

\footnotetext{
${ }^{7}$ We use the notation $\left[\chi_{S}\right] f$ to denote the Fourier coefficient of $\chi_{S}$ in the Fourier expansion of $f$.
}

In the case where $|S|$ is even, first consider the case where $w_{s}=$ $\gamma /|S|$ for $s \in S$. We then see that

$$
\left[\chi_{S}\right] \rho\left(w \cdot X_{S}+h\right)=\frac{2^{2 m}\left(2^{2 m}-1\right) B_{2 m}|S| !}{(2 m) !(2 m)|S|^{2 m}} \gamma^{2 m}+O\left(\gamma^{2 m+2}\right)
$$

and so as long as $\gamma$ is sufficiently small, the coefficient is positive. Next observe that if we flip the sign of $w_{s^{*}}$ for a single $s^{*} \in S$, then the sign of $\left[\chi_{S}\right]\left(w \cdot X_{S}+h\right)^{2 m}$ flips and so the sign of $\rho\left(w \cdot X_{S}+h\right)$ must also flip when $\gamma$ is sufficiently small. Since this coefficient varies continuously as a function of $w_{s^{*}}$, we see by the intermediate value theorem we see that we can get the coefficient of $\chi_{S}$ to be any value in $[-\delta, \delta]$ for some $\delta>0$.

The case when $|S|$ is odd is the same, except that we take $w_{s}=$ $\gamma /(|S|+1)$ and vary $h$ in $[-\gamma /(|S|+1), \gamma /(|S|+1)]$.

Proof of Theorem 4.4. By Lemma 4.3 this reduces to rewriting the MRF potential in term of a summation of $\rho(\cdot)$ terms coming from hidden units. We use the building block of Lemma B.1 and build the potential of the MRF in a top-down fashion. More precisely we can build any boolean function with Fourier mass supported on the first $r$ Fourier levels as follows:

(a) For each of the degree $r$ coefficients, use several copies of the parity building block to build a boolean function with the correct degree $r$ Fourier coefficients.

(b) Now recurse to the lower level coefficients - if we use only the building block for $|S| \leq r-1$ we will not affect the degree $r$ coefficients.

The end result is that any Markov random field of order $r$ can be converted into a Restricted Boltzmann distribution with hidden nodes of degree at most $r$, such that the observed nodes have the same distribution as the same Markov random field. If all of the Fourier coefficients of the potential of the original MRF are bounded by $M$, then the number of hidden units we need to introduce is $O_{d, M}(n)$, because given the upper bound on $d$ each visible unit is involved in only a constant number of hyperedges, and given the upper bound on $d$ and $M$ it takes only a constant number of copies of the building block to build each Fourier coefficient.

\section{APPENDIX TO SECTION 5}

\section{C.1 Examples of Over-parameterization}

Example C.1. Consider the Restricted Boltzmann machine with two observable nodes $\{1,2\}$ and two hidden nodes labeled $\{3,4\}$ such that $J_{13}=1, J_{23}=1$ and $J_{14}=-1, J_{24}=1$. Then the marginal distribution on the observables is just independent Rademachers, so this Restricted Boltzmann machine is not distinguishable from a model with no connections at all.

The previous example used non-ferromagnetic interactions to demonstrate the over-parameterization of RBMs. However, even when the RBM is ferromagnetic the model remains heavily overparameterized:

Example C.2. Consider a model with two observable nodes $\{1,2\}$, no external fields, and any number of hidden units/connections. Since the marginal distribution on $X_{1}$ and $X_{2}$ each must be Rademacher by symmetry, the observable distribution is specified just by a single parameter, the covariance between $X_{1}$ and $X_{2}$. However even in the simplest case, where there is only a single hidden unit connected 
to both $X_{1}$ and $X_{2}$, there are two parameters in the model, the two edge weights and we clearly see that these edge weights are not uniquely determined by the distribution.

Example C.3 (Hidden Structure is Undetermined). Consider an RBM with three observable nodes $\{1,2,3\}$, a single hidden node connected to all of them with positive edge weights, and no external field. We know the observable distribution is an MRF so it is of the form

$$
\operatorname{Pr}(X=x)=\frac{1}{Z} \exp \left(J_{12} x_{1} x_{2}+J_{13} x_{1} x_{3}+J_{23} x_{2} x_{3}+J_{123} x_{1} x_{2} x_{3}\right) .
$$

Perhaps surprisingly, in this model $J_{123}=0$. This can be seen from Lemma 4.3 and Taylor-expanding $\rho$, or simply by symmetry: the observable distribution is symmetric under the sign flip $x \mapsto$ $-x$ and so necessarily $J_{123}=0$. However, since there are only pairwise interactions in the potential it is easy to see (or we can apply Theorem 4.4) that there exists another RBM with only degree2 hidden nodes that has exactly the same observable distribution.

\section{C.2 Maximal Coefficients can be Arbitrarily Small}

In this subsection, we discuss some important obstacles to directly using regression-based methods (in particular [24]) for learning the parameters of a ferromagnetic RBM. By Lemma 4.3, we can cast the problem of learning $\mathcal{N}_{2}(i)$ for each node $i$ as a structure learning problem on the induced MRF. In order to use the results of Klivans and Meka [24], we need to get bounds on the potential

$$
p(x)=\sum_{j=1}^{m} \rho\left(w_{j} \cdot x+h_{j}^{(2)}\right)+\sum_{i=1}^{n} h_{i}^{(1)} x_{i} .
$$

In particular we need a bound on the size of the coefficients of $\partial_{i} p$. For a function $p:\{ \pm 1\}^{n} \rightarrow \mathbb{R}$, let $\|p\|_{1}$ denote the sum of the absolute values of its Fourier coefficients. Observe that

$$
\begin{aligned}
\left|\underset{X \sim\{ \pm 1\}^{n}}{\mathbf{E}}\left[\partial_{i} p \prod_{i \in S} X_{i}\right]\right| \\
\quad \leq\left|h_{i}^{(1)}\right|+\left|\underset{X \sim\{ \pm 1\}^{n}}{\mathbf{E}}\left[\sum_{j: w_{i j} \neq 0} \rho\left(w_{j} \cdot X+h_{j}^{(2)}\right) \prod_{i \in S} X_{i}\right]\right| \\
\quad \leq\left|h_{i}^{(1)}\right|+2 \beta \operatorname{deg}(i) \\
\quad \leq 2 \beta(\operatorname{deg}(i)+1)
\end{aligned}
$$

which follows from Holder's inequality, since $\left|\rho\left(w_{j} \cdot X+h_{j}^{(2)}\right)\right| \leq 2 \beta$ and $\left|h_{i}^{(1)}\right| \leq \beta$. Furthermore the coefficient of $X_{S}$ in $\partial_{i} p$ can be nonzero only when $S$ is a subset of the two-hop neighborhood of $i$, which follows from the Markov property. Thus we conclude that

$$
\left\|\partial_{i} p\right\|_{1} \leq 2^{d_{2}+1} \beta(\operatorname{deg}(i)+1)
$$

where $d_{2}$ is the maximum size of a node's two-hop neighborhood.

With this calculation in hand, the algorithm of Klivans and Meka [24] is able to estimate the maximal Fourier coefficients ${ }^{8}$ of the potential $p(x)$ to within $\epsilon$ additive error using roughly

$$
\frac{e^{O\left(d_{H} 2^{d_{2}+1} \beta\left(d_{V}+1\right)\right)}}{\epsilon^{4}} \log n
$$

${ }^{8}$ The guarantee [24] for recovering non-maximal coefficients is much weaker; in the full version of this paper, we give an example showing this is forced by informationtheoretic constraints if we only assume an $\ell_{1}$-constraint. samples where $d_{H}$ is the maximum degree of any hidden node and $d_{V}$ is the maximum degree of any observed node. We could then apply Theorem 7.2 of [24] to learn the two-hop neighborhoods in the RBM if we had an additional assumption that the induced MRF was $\eta$-identifiable:

Definition C.4. A Markov Random Field is $\eta$-identifiable if every maximum Fourier coefficent of its potential $p$ has magnitude at least $\eta$.

Unfortunately, even for MRFs induced by ferromagnetic RBMs and even under the assumption of $(\alpha, \beta)$-nondegeneracy, $\eta$ can be made to be arbitrarily small, as the following example shows:

Example C.5 (Failure of $\eta$-identifiability in ferromagnetic RBMs). Consider an RBM on three observed nodes with spins $X_{1}, X_{2}, X_{3}$ and a single hidden node with spin $Y_{1}$ connected to all of the observed nodes with edge weight $1 / 4$. On the hidden node let there be an external field $h_{1}^{(2)}=\epsilon$. When $\epsilon=0$, we see (as in Example C.3) that

$$
\operatorname{Pr}(X=x)=\frac{1}{Z} \exp \left(J X_{1} X_{2}+J X_{1} X_{3}+J X_{2} X_{3}\right)
$$

for some constant $J$ that is bounded away from zero. Hence the model is $\eta$-identifiable. However, for a small $\epsilon>0$, one can see by Taylor series expansion that the coefficient of $X_{1} X_{2} X_{3}$ is nonzero, and by continuity it can be made arbitrarily small by decreasing $\epsilon$. This does not affect the $(\alpha, \beta)$-nondegeneracy of the model, but clearly the parameter $\eta$ in $\eta$-identifiability goes to zero.

Thus existing guarantees for regression-based methods do not seem to be strong enough for our purposes. Moreover they would even require time $n^{d_{H}+1}$ to run, where $d_{H}$ is the hidden degree, since they solve a high-dimensional regression problem in the basis of all size $d_{H}$ monomials. In contrast our approach for learning the two-hop neighborhoods not only works in spite of the fact that the maximal Fourier coefficients can be arbitrarily small, it also runs in nearly quadratic time (see Theorem 6.1).

\section{C.3 Omitted Proofs}

In this subsection we give the omitted proofs for the hardness of learning RBMs. For the proofs purpose it is useful to recall the following equivalent (see Remark C.7) formulation of learning sparse parities as a hypothesis testing problem:

Definition C.6. The hypothesis testing problem for $k$-sparse parity with noise is to distinguish with high probability ${ }^{9}$ between the cases where $(X, Y)$ is drawn from the uniform distribution on $\{ \pm 1\}^{n+1}$ and where $(X, Y)$ is drawn from the $k$-sparse parity with noise distribution for an unknown $S$.

Remark C.7. It is clear that if we have an algorithm for the learning problem, we can use it for the hypothesis testing problem (the algorithm will return some set $S$ and we just have to test if the parity of $X_{S}$ is correlated with $Y$ ). In the other direction, observe that if we pick a particular $i$ and look at the marginal distribution on $\left(X_{\neq i}, Y\right)$ then if $i \in S$ this marginal distribution becomes uniform on $\{ \pm 1\}^{n}$, whereas if $i \notin S$ this is just a sparse parity with noise on a smaller number of variables, so if we can hypothesis test we can efficiently determine for every $i$ whether $i$ lies in $S$.

\footnotetext{
${ }^{9}$ i.e. with probability of Type I and Type II error going to 0 sufficiently fast.
} 
We now use this to show hardness for improper learning.

First we show hardness in the case of algorithms returning a distribution $Q$ with an (approximately) computable probability mass function.

Theorem C.8. If $k$-sparse parity with noise on $n$ bits cannot be learned in time $n^{o(k)}$, then there is no algorithm that runs in time $n^{o\left(d_{H}\right)} \cdot \operatorname{poly}(1 / \epsilon)$ and returns a probability distribution $Q$ such that:

(1) It is possible to (approximately) compute the $\operatorname{pmfQ}(x, y)$ for $x, y \in\{ \pm 1\}^{n} \times\{ \pm 1\}$ in polynomial time.

(2) $\|Q-\mathcal{P}\|_{\mathrm{TV}}<\epsilon$ where $\mathcal{P}$ is the distribution on the observables of an $R B M$ with hidden degree $d_{H}$.

Proof of Theorem C.8. We show how to use $Q$ to solve the hypothesis testing problem for sparse parity with noise. Recall that for any distributions $\mathcal{P}_{1}, \mathcal{P}_{2}$

$$
\left\|\mathcal{P}_{1}-\mathcal{P}_{2}\right\|_{\mathrm{TV}}=\underset{X \sim \mathcal{P}_{1}}{\mathbf{E}}\left[\frac{\mathcal{P}_{1}(X)-\mathcal{P}_{2}(X)}{\mathcal{P}_{1}(X)} \mathbf{1}\left[\mathcal{P}_{1}(X) \geq \mathcal{P}_{2}(X)\right]\right]
$$

and observe that the quantity inside the expectation is always valued in $[0,1]$. Therefore, with $\mathcal{P}_{1}=\operatorname{Unif}\left(\{ \pm 1\}^{\mathrm{n}+1}\right)$ and $\mathcal{P}_{2}=Q$, we may use $m$ samples from $\mathcal{P}_{1}$ and the above formula to approximate the TV between $Q$ and the uniform distribution on $\{ \pm 1\}^{n+1}$ within error $O(1 / \sqrt{m})$ with high probability (by Hoeffding's inequality). Since the TV distance between the uniform distribution and any particular sparse parity with noise is $\Omega(\eta)$ (consider the tester that looks at whether $Y=\prod_{s \in S} X_{s}$ ), this lets us solve the hypothesis testing problem for sparse parity with noise. Thus, if the algorithm can find $Q$ in time $n^{o\left(d_{H}\right)}$, then this violates the conjectured hardness of learning sparse parity with noise.

We now prove the result in the case we have access to an unnormalized pmf.

Proof of Theorem 5.5. We again reduce from the hypothesis testing problem for sparse parity with noise. As before suppose $Z^{(1)}, \ldots, Z^{(m)}$ are iid samples from the uniform distribution on $\{ \pm 1\}^{n+1}$; we will look at the statistics of $q(Z)$. Observe that if $Q$ were the uniform distribution, then we would have $q(Z)=$ $C_{q} 1 / 2^{n+1}$, whereas if $Q$ were a sparse parity with noise we would have $q(Z) \propto e^{J_{\eta} \prod_{s \in S} Z_{s}}$ where $J_{\eta}$ is a constant that corresponds to $\eta$.

Let $q_{1 / 3}$ be such that the number of $z^{(i)}$ with $q\left(Z^{(i)}\right) \leq q_{1 / 3}$ is at most $m / 3$, and define $q_{2 / 3}$ similarly. Consider the quantity $V:=\frac{q_{2 / 3}-q_{1 / 3}}{q_{1 / 3}+q_{2 / 3}}$. Under the uniform distribution $V$ is concentrated around zero, whereas under a sparse parity distribution $V$ is concentrated about $\frac{e^{J_{\eta}}-e^{-J_{\eta}}}{e^{J_{\eta}}+e^{-J_{\eta}}}$. The same is true under distributions which are close in TV to either distribution, since $V$ is defined in terms of cumulative distribution function statistics. Therefore we can distinguish between independent bits and sparse parity with noise efficiently given access to $q$.

\section{APPENDIX TO SECTION 6}

\section{D.1 Analysis of GreEDYNBHD}

$(\alpha, \beta)$-nondegeneracy has the following useful consequences:
Lemma D.1. Suppose $X_{i}$ is the spin at vertexi in an $(\alpha, \beta)$-nondegenerate Ising model. Then $\min \left(\operatorname{Pr}\left(X_{i}=+\right), \operatorname{Pr}\left(X_{i}=-\right)\right) \geq \sigma(-2 \beta)$, where $\sigma(x)=\frac{1}{1+e^{-x}}$.

Proof. We show the lower bound for $\operatorname{Pr}\left(X_{i}=+\right)$ since the two cases are symmetrical. By the law of total expectation, it suffices to show that for any fixing $x_{\neq i}$ of the other spins $X_{\neq i}$ that $\operatorname{Pr}\left(X_{i}=\right.$ $\left.+\mid X_{\neq i}=x_{\neq i}\right) \geq \sigma(-2 \beta)$, and this follows because

$$
\begin{aligned}
\operatorname{Pr}\left(X_{i}=+\mid X_{\neq i}=x_{\neq i}\right) & =\frac{\exp \left(\sum_{j: j \neq i} J_{i j} x_{j}\right)}{\exp \left(\sum_{j: j \neq i} J_{i j} x_{j}\right)+\exp \left(-\sum_{j: j \neq i} J_{i j} x_{j}\right)} \\
& =\sigma\left(2 \sum_{j: j \neq i} J_{i j} x_{j}\right) \geq \sigma(-2 \beta) .
\end{aligned}
$$

Lemma D.2. Suppose that $X_{i}$ is the spin at vertex $i$ in an $(\alpha, \beta)$ nondegenerate Ising model and $j$ is a neighbor of $i$. Then for any fixing $x_{\neq i, j}$ of the other spins $X_{i \neq j}$ of the Ising model, we have

$$
\begin{aligned}
& \left|\mathbf{E}\left[X_{i} \mid X_{j}=1, X_{\neq i, j}=x_{\neq i, j}\right]-\mathbf{E}\left[X_{i} \mid X_{j}=-1, X_{\neq i, j}=x_{\neq i, j}\right]\right| \\
& \quad \geq 2 \alpha\left(1-\tanh ^{2}(\beta)\right) .
\end{aligned}
$$

Proof. Observe that $\mathrm{E}\left[X_{i} \mid X_{\neq i}\right]=\tanh \left(\sum_{k: k \neq i} J_{i k} x_{k}\right)$. Since $\tanh ^{\prime}(x)=1-\tanh ^{2}(x)$ and tanh is a monotone function, we see that if we let $x=-J_{i j}+\sum_{k: k \notin\{i, j\}} J_{i k} x_{k}$, then since $x \in[-\beta, \beta]$ we have

$$
\begin{aligned}
\left|\tanh \left(x+2 J_{i j}\right)-\tanh (x)\right| & \geq 2\left|J_{i j}\right| \inf _{x \in[-\beta, \beta]}\left(1-\tanh ^{2}(x)\right) \\
& \geq 2 \alpha\left(1-\tanh ^{2}(\beta)\right) .
\end{aligned}
$$

The following lemma shows quantitatively that in a nondegenerate ferromagnetic RBM, the graph-theoretic two-hop neighborhood of a vertex $i$ always equals $\mathcal{N}_{2}(i)$, the two-hop Markov blanket. It is immediate from the Markov property for the RBM as an Ising model that $\mathcal{N}_{2}(i)$ is contained in the graph-theoretic two-hop neighborhood, and the lemma implies the reverse inclusion.

Lemma D.3. Suppose node $i$ is an observed node in a ferromagnetic $(\alpha, \beta)$-nondegenerate RBM and denote by $T$ the graph-theoretic twohop neighborhood of $i$. If $S \subset[n]$ is a set of nodes such that $T \not \subset S$, then for any $j \in T \backslash S$, we have

$$
I_{i}(S \cup\{j\})-I_{i}(S) \geq 2 \alpha^{2} \sigma(-2 \beta)(1-\tanh (\beta))^{2} .
$$

Proof. Fix $j \in \mathcal{N}_{\in}(i) \backslash S$ and let $k$ be a hidden node which is a mutual neighbor of $i, j$. Now observe by submodularity it suffices to prove the lower bound when $S=[n] \backslash\{i, j, k\}$. Then

$$
\begin{aligned}
& I_{i}(S \cup\{j\})-I_{i}(S) \\
&=\mathbf{E}\left[X_{i} \mid X_{S}=1^{S}, X_{j}=1\right]-\mathbf{E}\left[X_{i} \mid X_{S}=1^{S}\right] \\
&= \mathbf{E}\left[X_{i} \mid X_{S}=1^{S}, X_{j}=1\right] \\
&-\mathbf{E}\left[X_{i} \mid X_{S}=1^{S}, X_{j}=1\right] \operatorname{Pr}\left(X_{j}=1 \mid X_{S}=1^{S}\right) \\
&-\mathbf{E}\left[X_{i} \mid X_{S}=1^{S}, X_{j}=-1\right] \operatorname{Pr}\left(X_{j}=-1 \mid X_{S}=1^{s}\right) \\
&= \operatorname{Pr}\left(X_{j}=-1 \mid X_{S}=1^{S}\right)\left(\mathbf{E}\left[X_{i} \mid X_{S}=1^{S}, X_{j}=1\right]\right. \\
&\left.-\mathbf{E}\left[X_{i} \mid X_{S}=1^{S}, X_{j}=-1\right]\right) \\
& \geq \sigma(-2 \beta)\left(\mathbf{E}\left[X_{i} \mid X_{S}=1^{S}, X_{j}=1\right]-\mathbf{E}\left[X_{i} \mid X_{S}=1^{S}, X_{j}=-1\right]\right) .
\end{aligned}
$$


Furthermore when $S=[n] \backslash\{i, j, k\}$ we know that $X_{i}$ and $X_{j}$ are independent conditioned on $k$, so

$$
\begin{aligned}
\mathbf{E} & {\left[X_{i} \mid X_{S}=1^{S}, X_{j}=1\right]-\mathbf{E}\left[X_{i} \mid X_{S}=1^{S}, X_{j}=-1\right] } \\
= & \mathbf{E}\left[X_{i} \mid X_{S}=1^{S}, X_{k}=1\right]\left(\operatorname{Pr}\left(X_{k}=1 \mid X_{S}=1^{S}, X_{j}=1\right]\right. \\
& \left.-\operatorname{Pr}\left(X_{k}=1 \mid X_{S}=1^{S}, X_{j}=-1\right]\right) \\
& +\mathrm{E}\left[X_{i} \mid X_{S}=1^{S}, X_{k}=-1\right]\left(\operatorname{Pr}\left(X_{k}=-1 \mid X_{S}=1^{S}, X_{j}=1\right]\right. \\
& \left.-\operatorname{Pr}\left(X_{k}=-1 \mid X_{S}=1^{S}, X_{j}=-1\right]\right) \\
= & \mathbf{E}\left[X_{i} \mid X_{S}=1^{S}, X_{k}=1\right]\left(\operatorname{Pr}\left(X_{k}=1 \mid X_{S}=1^{S}, X_{j}=1\right]\right. \\
& \left.-\operatorname{Pr}\left(X_{k}=1 \mid X_{S}=1^{S}, X_{j}=-1\right]\right) \\
& -\mathbf{E}\left[X_{i} \mid X_{S}=1^{S}, X_{k}=-1\right]\left(\operatorname{Pr}\left(X_{k}=1 \mid X_{S}=1^{S}, X_{j}=1\right]\right. \\
& \left.-\operatorname{Pr}\left(X_{k}=1 \mid X_{S}=1^{S}, X_{j}=-1\right]\right) \\
= & \left(\mathbf{E}\left[X_{i} \mid X_{S}=1^{S}, X_{k}=1\right]-\mathbf{E}\left[X_{i} \mid X_{S}=1^{S}, X_{k}=-1\right]\right) \\
& \cdot\left(\operatorname{Pr}\left(X_{k}=1 \mid X_{S}=1^{S}, X_{j}=1\right]-\operatorname{Pr}\left(X_{k}=1 \mid X_{S}=1^{S}, X_{j}=-1\right]\right) \\
\geq & 2 \alpha^{2}(1-\tanh (\beta))^{2},
\end{aligned}
$$

where the last inequality is by Lemma D.2.

As the first step in analyzing our algorithm, we first determine a sufficient number of samples to compute $\widetilde{I}_{i}(S)$ to a specified precision for all small sets $S$.

Lemma D.4. Let $\delta, \epsilon>0$ and $k \geq 0$. If we are given $M$ samples from a ferromagnetic Restricted Boltzmann Machine and $M \geq$ $2^{2 k+1}\left(1 / \epsilon^{2}\right)(\log (n)+k \log (e n / k)) \log (4 / \delta)$, then with probability at least $1-\delta$, for all $S \subset[n]$ such that $|S| \leq k,\left|I_{i}(S)-\widetilde{I}_{i}(S)\right|<\epsilon$.

Proof of Lemma D.4. First observe that

$$
\operatorname{Pr}\left(X_{S}=1^{S}\right) \geq 2^{-|S|}
$$

because in a ferromagnetic model (which by our definition has nonnegative external fields), $X_{S}=1^{S}$ is the most likely state to observe for $X_{S}$. This inequality can also be proved by applying Griffith's inequality iteratively. Also observe that the total number of sets $S$ we consider is $\sum_{j=0}^{k}\left(\begin{array}{c}n \\ j\end{array}\right) \leq(e n / k)^{k}$. For each $S$, let $M_{S}$ be the number of samples where $X_{S}=1^{S}$. Then by Hoeffding's inequality, $\operatorname{Pr}\left(M_{S}-\mathrm{E} M_{S}<-t\right) \leq e^{-2 t^{2} / M}$. In particular, since E $M_{S} \geq 2^{-k} M$ as long as $|S| \leq k, \operatorname{Pr}\left(M_{S}<2^{-k-1} M\right) \leq e^{-2 M 2^{-2 k-2}}$.

Now by the usual rejection sampling argument, those samples which have $X_{S}=1^{S}$ are independent and identically distributed samples from the conditional law. (One way to see this is that we can think of each sample as equivalently being generated by first sampling $X_{S}$, then sampling the rest of the spins conditioned on $X_{S}$ ). Therefore, by another application of Hoeffding's inequality, for a particular choice of $i, S$ we have

$$
\operatorname{Pr}\left(\left|\widetilde{I}_{i}(S)-I_{i}(S)\right| \geq \epsilon \mid M_{S}\right) \leq 2 e^{-2 M_{S} \epsilon^{2}}
$$

Now by the law of total expectation

$$
\begin{aligned}
\operatorname{Pr}\left(\left|\widetilde{I}_{i}(S)-I_{i}(S)\right| \geq \epsilon\right) & =\mathrm{E}\left[\operatorname{Pr}\left(\left|\widetilde{I}_{i}(S)-I_{i}(S)\right| \geq \epsilon \mid M_{S}\right)\right] \\
& \leq 2 \mathrm{E}\left[e^{-2 M_{S} \epsilon^{2}}\right] \\
& =2 \mathbf{E}\left[\left(\mathbf{1}_{M_{S}<2^{-k-1} M}+\mathbf{1}_{M_{S} \geq 2^{-k-1} M}\right) e^{-2 M_{S} \epsilon^{2}}\right] \\
& \leq 2 e^{-2 M 2^{-2 k-2}}+2 e^{-2\left(2^{-k-1} M\right) \epsilon^{2}} \\
& \leq 4 e^{-M 2^{-2 k-1} \epsilon^{2}} .
\end{aligned}
$$

And by the union bound, the probability that $\left|\widetilde{I}_{i}(S)-I_{i}(S)\right| \geq \epsilon$ for some $i, S$ is at most $n(e n / k)^{k} 4 e^{-M 2^{-2 k-1} \epsilon^{2}}$. Therefore if we take $M \geq 2^{2 k+1}\left(1 / \epsilon^{2}\right)(\log (n)+k \log (e n / k)) \log (4 / \delta)$ the result follows.

We also analyze the standard greedy algorithm for submodular maximization under noise; this corresponds to Steps 1-2 of the algorithm.

Lemma D.5. Suppose $t \geq 0$ is an integer, $f(S)$ is a monotone submodular function and $\widetilde{f}(S)$ is an approximation to $f$ such that $|f(S)-\widetilde{f}(S)|<\epsilon$ for some uniform $\epsilon>0$ and all $S$ such that $|S| \leq t$. Let $S_{0}=\emptyset$ and suppose $S_{i+1}$ is formed by greedily adding to $S_{i}$ the element $j$ which maximizes $\widetilde{f}\left(S_{i} \cup\{j\}\right)$. Then for any set $T$, we have

$$
f(T)-f\left(S_{t}\right) \leq(1-1 /|T|)^{t} f(T)+|T| \epsilon .
$$

Proof. Consider going from $S_{t}$ to $S_{t+1}$. By Lemma 3.7, there exists some $j^{*}$ such that

$$
f\left(S_{t} \cup\left\{j^{*}\right\}\right)-f\left(S_{t}\right) \geq \frac{f(T)-f\left(S_{t}\right)}{|T|} .
$$

Therefore for the $j$ which is chosen to form $S_{t+1}$, we know

$$
\begin{aligned}
\left(f(T)-f\left(S_{t}\right)\right)-\left(f(T)-f\left(S_{t+1}\right)\right) & =f\left(S_{t+1}\right)-f\left(S_{t}\right) \\
& =f\left(S_{t} \cup\{j\}\right)-f\left(S_{t}\right) \\
& \geq \frac{f(T)-f\left(S_{t}\right)}{|T|}-\epsilon .
\end{aligned}
$$

Rearranging, we see that

$$
f(T)-f\left(S_{t+1}\right) \leq(1-1 /|T|)\left(f(T)-f\left(S_{t}\right)\right)+\epsilon
$$

and the result follows by iterating this inequality (note that the sum of the epsilon terms forms a geometric series).

Proof of Theorem 6.1. Apply Lemma D.4 with $\epsilon=\eta /\left(4 d_{2}\right)$; then for our choice of $M$ we have that $\left|\widetilde{I}_{i}(S)-I_{i}(S)\right|<\eta /\left(4 d_{2}\right)$ for all $S$ with $|S| \leq k$. Then applying Lemma D.5 and using our choice of $k$ with the inequality $1+x \leq e^{x}$, we have

$$
I_{i}\left(\mathcal{N}_{2}(i)\right)-I_{i}\left(S_{k}\right) \leq\left(1-1 / d_{2}\right)^{k}+\eta / 4 \leq \eta / 2 .
$$

Suppose $S_{k}$ does not contain the two-hop neighborhood of $i$. then we can take any of the two-hop neighbors $j \in \mathcal{N}_{2}(i) \backslash S_{k}$ and see that

$$
\begin{aligned}
I_{i}\left(\mathcal{N}_{2}(i)\right)-I_{i}\left(S_{k}\right) & \geq I_{i}\left(S_{k} \cup\{j\}\right)-I_{i}\left(S_{k}\right) \\
& \geq 2 \alpha^{2} \sigma(-2 \beta)(1-\tanh (\beta))^{2}=2 \eta
\end{aligned}
$$

where the first inequality follows since $\mathcal{N}_{2}(i)$ is the global maximizer of $I_{i}$ among all subsets of the observed nodes (by monotonicity and the Markov property), and the second inequality is Lemma D.3. 
This contradicts (1), therefore $S_{k}$ does contain the entire two-hop neighborhood of $i$.

It remains to show that Step 3 of the algorithm leaves in $\widetilde{\mathcal{N}}_{2}$ exactly the elements of $S$ which are in the two-hop neighborhood. Since $\left|\widetilde{I}_{i}(S)-I_{i}(S)\right|<\eta /\left(4 d_{2}\right)$ for every set $S$ with $|S| \leq k$, this is straightforward: if $j$ is a two-hop neighbor, then by Lemma D.3 and triangle inequality we see that

$$
\left|\widetilde{I}_{i}\left(S_{k}\right)-\widetilde{I}_{i}\left(S_{k} \backslash\{j\}\right)\right| \geq 2 \eta-\eta / 2>\eta
$$

If $j$ is not a two-hop neighbor, then $I_{i}\left(S_{k}\right)-I_{i}\left(S_{k} \backslash\{j\}\right)=0$ by the Markov property, so by triangle inequality $\left|\widetilde{I}_{i}\left(S_{k}\right)-\widetilde{I}_{i}\left(S_{k} \backslash\{j\}\right)\right| \leq$ $\eta / 2<\eta$. Thus for each $i$, the returned $\widetilde{\mathcal{N}}_{2}$ is the true two-hop neighborhood of vertex $i$.

To analyze the runtime, observe that the loop goes through at most $k$ steps, and each iteration of the loop takes time $O(n M)$ to consider each $j$ and compute $\widetilde{I}\left(S_{t} \cup\{j\}\right)$ from samples, and we run GREEDYNBHD from each of the $n$ vertices.

\section{ACKNOWLEDGMENTS}

We are grateful to Elchanan Mossel for valuable discussions about this work and to Linus Hamilton for valuable preliminary discussions. We also thank Raghu Meka for useful feedback.

\section{REFERENCES}

[1] Animashree Anandkumar, Vincent YF Tan, Furong Huang, and Alan S Willsky. 2012. High-dimensional structure estimation in Ising models: Local separation criterion. The Annals of Statistics (2012), 1346-1375.

[2] Animashree Anandkumar and Ragupathyraj Valluvan. 2013. Learning loopy graphical models with latent variables: Efficient methods and guarantees. The Annals of Statistics (2013), 401-435.

[3] Sanjeev Arora, Aditya Bhaskara, Rong Ge, and Tengyu Ma. 2014. Provable bounds for learning some deep representations. In International Conference on Machine Learning. 584-592.

[4] Alexander Barvinok. 2016. Computing the permanent of (some) complex matrices. Foundations of Computational Mathematics 16, 2 (2016), 329-342.

[5] Andrej Bogdanov, Elchanan Mossel, and Salil Vadhan. 2008. The complexity of distinguishing Markov random fields. In Approximation, Randomization and Combinatorial Optimization. Algorithms and Techniques. Springer, 331-342.

[6] Guy Bresler. 2015. Efficiently learning Ising models on arbitrary graphs. In Proceedings of the Forty-Seventh Annual ACM on Symposium on Theory of Computing. ACM, 771-782.

[7] Guy Bresler, David Gamarnik, and Devavrat Shah. 2014. Hardness of parameter estimation in graphical models. In Advances in Neural Information Processing Systems. 1062-1070.

[8] Guy Bresler, Elchanan Mossel, and Allan Sly. 2008. Reconstruction of Markov random fields from samples: Some observations and algorithms. In Approxima tion, Randomization and Combinatorial Optimization. Algorithms and Techniques. Springer, 343-356.

[9] Gruia Calinescu, Chandra Chekuri, Martin Pál, and Jan Vondrák. 2007. Maximizing a submodular set function subject to a matroid constraint. In International Conference on Integer Programming and Combinatorial Optimization. Springer 182-196.

[10] Venkat Chandrasekaran, Pablo A Parrilo, and Alan S Willsky. 2012. Latent variable graphical model selection via convex optimization. The Annals of Statistics 40, 4 (2012), 1935-1967.

[11] C Chow and Cong Liu. 1968. Approximating discrete probability distributions with dependence trees. IEEE transactions on Information Theory 14, 3 (1968), 462-467.

[12] Adam Coates, Andrew Ng, and Honglak Lee. 2011. An analysis of single-layer networks in unsupervised feature learning. In Proceedings of the fourteenth international conference on artificial intelligence and statistics. 215-223.

[13] Ronen Eldan and Ohad Shamir. 2016. The power of depth for feedforward neural networks. In Conference on Learning Theory. 907-940.

[14] Surbhi Goel and Adam Klivans. 2017. Learning depth-three neural networks in polynomial time. arXiv preprint arXiv:1709.06010 (2017).
[15] Robert B. Griffiths, C. A. Hurst, and S. Sherman. 1970. Concavity of Magnetization of an Ising Ferromagnet in a Positive External Field. F. Math. Phys. 11, 3 (1970), 790795. https://doi.org/10.1063/1.1665211 arXiv:https://doi.org/10.1063/1.1665211

[16] Linus Hamilton, Frederic Koehler, and Ankur Moitra. 2017. Information theoretic properties of Markov random fields, and their algorithmic applications. In Advances in Neural Information Processing Systems. 2460-2469.

[17] Geoffrey E Hinton. 2009. Deep belief networks. Scholarpedia 4, 5 (2009), 5947.

[18] Geoffrey E Hinton and Ruslan R Salakhutdinov. 2006. Reducing the dimensionality of data with neural networks. science 313, 5786 (2006), 504-507.

[19] Geoffrey E Hinton and Ruslan R Salakhutdinov. 2009. Replicated softmax: an undirected topic model. In Advances in neural information processing systems. $1607-1614$.

[20] Majid Janzamin, Hanie Sedghi, and Anima Anandkumar. 2015. Beating the perils of non-convexity: Guaranteed training of neural networks using tensor methods. arXiv preprint arXiv:1506.08473 (2015).

[21] M. Jerrum and A. Sinclair. 1990. Polynomial-Time Approximation Algorithms for Ising Model (Extended Abstract). In Automata, Languages and Programming. $462-475$.

[22] Sham M Kakade, Varun Kanade, Ohad Shamir, and Adam Kalai. 2011. Efficient learning of generalized linear and single index models with isotonic regression. In Advances in Neural Information Processing Systems. 927-935.

[23] David Karger and Nathan Srebro. 2001. Learning Markov networks: Maximum bounded tree-width graphs. In Proceedings of the twelfth annual ACM-SIAM symposium on Discrete algorithms. Society for Industrial and Applied Mathematics, 392-401.

[24] Adam Klivans and Raghu Meka. 2017. Learning Graphical Models Using Multiplicative Weights. In FOCS.

[25] Tsung-Dao Lee and Chen-Ning Yang. 1952. Statistical theory of equations of state and phase transitions. II. Lattice gas and Ising model. Physical Review 87, 3 (1952), 410.

[26] Jingcheng Liu, Alistair Sinclair, and Piyush Srivastava. 2017. The Ising Partition Function: Zeros and Deterministic Approximation. 2017 IEEE 58th Annual Symposium on Foundations of Computer Science (FOCS) (2017), 986-997.

[27] Philip M Long and Rocco Servedio. 2010. Restricted Boltzmann machines are hard to approximately evaluate or simulate. In Proceedings of the 27th International Conference on Machine Learning (ICML-10). 703-710.

[28] Christopher W Lynn and Daniel D Lee. 2018. Maximizing Activity in Ising Networks via the TAP Approximation. arXiv preprint arXiv:1803.00110 (2018).

[29] James Martens, Arkadev Chattopadhya, Toni Pitassi, and Richard Zemel. 2013. On the representational efficiency of restricted boltzmann machines. In Advances in Neural Information Processing Systems. 2877-2885.

[30] Elchanan Mossel, Ryan O'Donnell, and Rocco P Servedio. 2003. Learning juntas. In Proceedings of the thirty-fifth annual ACM symposium on Theory of computing. ACM, 206-212.

[31] George L Nemhauser, Laurence A Wolsey, and Marshall L Fisher. 1978. An analysis of approximations for maximizing submodular set functionsâĂŤI. Mathematical Programming 14, 1 (1978), 265-294.

[32] Pradeep Ravikumar, Martin J Wainwright, John D Lafferty, et al. 2010. Highdimensional Ising model selection using ?1-regularized logistic regression. The Annals of Statistics 38, 3 (2010), 1287-1319.

[33] Itay Safran and Ohad Shamir. 2017. Depth-width tradeoffs in approximating natural functions with neural networks. In International Conference on Machine Learning. 2979-2987.

[34] Ruslan Salakhutdinov, Andriy Mnih, and Geoffrey Hinton. 2007. Restricted Boltzmann machines for collaborative filtering. In Proceedings of the 24th international conference on Machine learning. ACM, 791-798.

[35] Narayana P Santhanam and Martin J Wainwright. 2012. Information-theoretic limits of selecting binary graphical models in high dimensions. IEEE Transactions on Information Theory 58, 7 (2012), 4117-4134.

[36] Allan Sly and Nike Sun. 2012. The computational hardness of counting in twospin models on d-regular graphs. In Foundations of Computer Science (FOCS), 2012 IEEE 53rd Annual Symposium on. IEEE, 361-369.

[37] Matus Telgarsky. 2016. Benefits of depth in neural networks. In Conference on Learning Theory. 1517-1539.

[38] Gregory Valiant. 2012. Finding correlations in subquadratic time, with applications to learning parities and juntas. In Foundations of Computer Science (FOCS), 2012 IEEE 53rd Annual Symposium on. IEEE, 11-20.

[39] Marc Vuffray, Sidhant Misra, Andrey Lokhov, and Michael Chertkov. 2016. Interaction screening: Efficient and sample-optimal learning of Ising models. In Advances in Neural Information Processing Systems. 2595-2603.

[40] Yuchen Zhang, Jason D Lee, and Michael I Jordan. 2016. $\ell_{1}$-regularized neural networks are improperly learnable in polynomial time. In International Conference on Machine Learning. 993-1001. 